\title{
A Model of Radiative Recombination in (In,Al,Ga)N/GaN Structures with Significant Potential Fluctuations
}

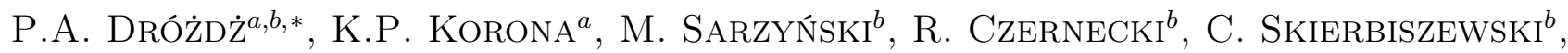 \\ G. MUZiǫ ${ }^{b}$ AND T. SUSKI ${ }^{b}$ \\ ${ }^{a}$ Faculty of Physics, University of Warsaw, L. Pasteura 5, 02-093 Warsaw, Poland \\ ${ }^{b}$ Institute of High Pressure Physics "Unipress", Polish Academy of Sciences, \\ Sokołowska 29/37, 01-142 Warsaw, Poland
}

\begin{abstract}
The potential fluctuations in III-nitride quantum wells lead to many effects like emission broadening and S-shape energy vs. temperature dependence. The best description of the energy dependence comes from calculations based on Gaussian density of states. However, in most of the published reports, changes of carrier lifetime with energy and temperature are not taken into account. Since experimental evidence shows that lifetime significantly depends on energy and temperature, here we propose a model that describes two basic parameters of luminescence: lifetime of carries and emission energy as a function of temperature in the case of quantum wells and layers that are characterized by potential fluctuations. Comparison of the measured energy and lifetime dependences on temperature in specially grown InGaN/GaN quantum wells and InAlGaN layer shows very good agreement with the proposed theoretical approach.
\end{abstract}

DOI: 10.12693/APhysPolA.130.1209

PACS/topics: 78.55.Cr, 73.22.-f, 78.47.jd

\section{Introduction}

Radiative recombination of excitons (or uncoupled electron-hole pairs) is the main physical phenomenon decisive for efficient light emission in light emitting diodes and laser diodes. For devices consisting of InGaN/GaN quantum wells (QWs) the recombination strongly depends on localization of carriers. This effect is also well pronounced in layers of ternary and quaternary nitride semiconductors. The inhomogeneous In-distribution and QW width fluctuations result in carrier localization effective in the low temperature and weak carrier excitation regimes. High enough temperatures and strong excitation of carriers cause their delocalization. Potential fluctuations have been observed by many authors. Beside of structural studies by means of transmission electron microscopy, optical measurements have been used quite often. Results obtained by the latter methods supply useful information via analysis of the emission energy $\left(E_{\mathrm{PL}}\right)$ shift with temperature and time. Standard thermal dependence of $E_{\mathrm{PL}}$ in pure semiconductors (binary alloys) behaves similarly to the band gap. It decreases monotonically with temperature and can be described by simple functions [1-3]. However, in structures made of alloys (like GaInN/GaN QW) the photoluminescence $(\mathrm{PL})$ energy dependence on temperature is more complicated [4-6]. During heating of the QW sample from cryogenic temperature region, $E_{\mathrm{PL}}$ decreases at first, than increases, and decreases again at high temperatures resulting in so called "S-shape". It is explained by potential fluctuations. At low temperatures, carriers

\footnotetext{
* corresponding author; e-mail: piotr.drozdz@fuw.edu.pl
}

relax to regions of lower potential. While the temperature is increased, carriers start to thermally escape, so $E_{\mathrm{PL}}$ obtains higher values. At higher temperatures the standard bandgap dependence on $T$ plays dominant role, i.e., $E_{\mathrm{PL}}$ decreases with $T$. Analysis of the S-shape dependence can give information about the amplitude and shape of potential fluctuations which is important for determination of the recombination effectiveness [7].

Several attempts have been made to describe thermal dependence of $E_{\mathrm{PL}}[8]$. They were based on assumption of some scattered density of states (DOS) in the fluctuating potential that is occupied by carriers according to the Boltzmann distribution [8] or more complicated dependence [9]. However, it was assumed that radiative recombination time is independent of temperature and energy. Due to the significant discrepancies between these assumptions and the experimental observations we decided to propose an improved approach to the problem of fluctuation-induced S-shape in temperature dependence of $E_{\mathrm{PL}}$.

\section{Theory}

The thermodynamic dependence of GaN band gap can be estimated by simple formula [3], however in order to describe this dependence in InGaN compound, fluctuations of indium content must be taken into account.

In order to describe thermal dependence of $E_{\mathrm{PL}}$ in the case of such fluctuations, Eliseev [8] proposed Gaussian density of states (DOS) of the form

$$
\rho(E)=\rho_{0} \exp \left(\frac{-\left(E-E_{0}\right)^{2}}{2 \sigma^{2}}\right)
$$

where $E_{0}$ is the central energy, and $\sigma$ is dispersion (see Fig. 1). It has been assumed that the carrier distribution function obeys the Boltzmann statistics $f(E, T)=$ 


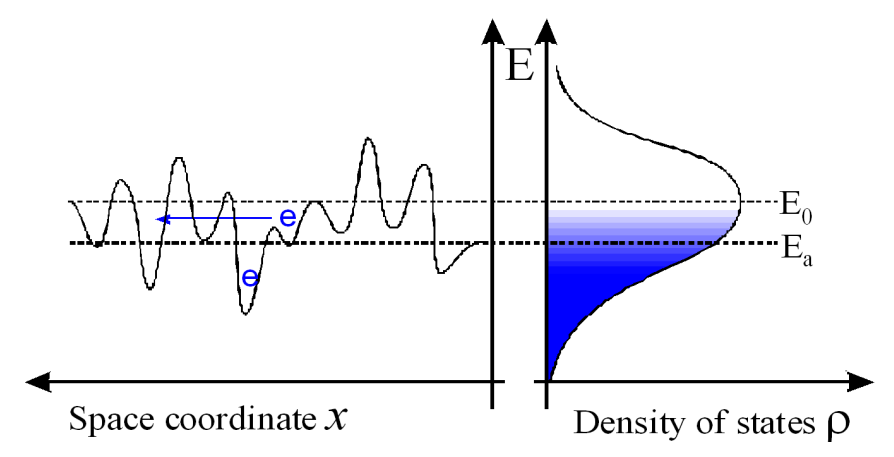

Fig. 1. Scheme of fluctuations that lead to the broad density of states $\rho$, centered at $E_{0} . E_{\text {a }}$ is delocalisation threshold.

$B \exp \left(-E / k_{\mathrm{B}} T\right)$. The energy of electron density maximum, $E_{\text {eff }}(T)$, is related to ideal material bandgap $E_{\mathrm{S}}(T)$, by factor $\varepsilon(T)$ :

$$
E_{\text {eff }}(T)=E_{\mathrm{S}}(T)-\varepsilon(T) k_{\mathrm{B}} T \text {. }
$$

Simple calculation gives $\varepsilon(T)=\left(\sigma / k_{\mathrm{B}} T\right)^{2}$ [8]. The Eliseev theory successfully describes energy dependence in high temperatures, however, it fails in lower temperatures.

The description in low temperature range was improved by $\mathrm{Li}$ et al. [9]. In the introduced model, thermal activation and the transfer of excitons/carriers localized at different potential minima was taken into account. Li and collaborators assumed that the trapping and escape are internal processes that do not change global number of particles. Moreover, these processes are in equilibrium, so the shape of the carrier distribution does not change with time. The number of particles decreases only by radiative recombination characterized by time $\tau_{\mathrm{r}}$. Both radiative recombination and thermal escape are assumed to be time and energy independent [9].

Such approach gives model that describes S-shape quite well, however, it neglects changes of recombination time with energy and temperature. Since experimental evidence points to significant thermal dependence of lifetime, here we propose an improved approach that gives proper recombination vs. temperature dependence. Since photons have very low momentum, emission of light by moving particle is suppressed by momentum conservation rule. The dependence of an average momentum and a radiative lifetime for $n$-dimensional objects should be proportional to $T^{n / 2}$ [10]. For 0-dimensional structures (localized particles), radiative recombination time should be constant [11], whereas for quantum wells linear dependence between radiative recombination time and temperature takes place. Since particles are distributed over many states, we must estimate the proportions of localized to delocalized particles. We assume that the fraction proportional to $\exp \left(\left(E_{\mathrm{a}}-E\right) / k_{\mathrm{B}} T\right)$ is delocalized. Therefore, we propose the following formula to approximate radiative time, $\tau_{\mathrm{r}}$, dependence on energy and temperature:

$$
\tau_{\mathrm{r}}(E, T)=\tau_{\mathrm{a}}+\alpha T \exp \left(\frac{E_{\mathrm{a}}-E}{k_{\mathrm{B}} T}\right),
$$

where $\tau_{\mathrm{a}}$ is the time constant, $E_{\mathrm{a}}$ is the energy of delocalization and $\alpha$ is a lifetime-temperature coefficient. For low temperatures $\tau_{\mathrm{r}}$ can be approximated by constant $\tau_{\mathrm{a}}$, whereas for high energy and temperatures, linear dependence becomes important.

Since recombination probability $\left(1 / \tau_{\mathrm{r}}\right)$ dependence on energy, it changes shape of emission peak. Position of the PL peak maximum can be found by calculation of the factor $\varepsilon(T)$ by solving of the following expression:

$$
\frac{\alpha T}{\tau_{\mathrm{a}}} \mathrm{e}^{-\varepsilon} \mathrm{e}^{\frac{-\Delta E}{k_{\mathrm{B}} T}}=\frac{\varepsilon-\left(\frac{\sigma}{k_{\mathrm{B}} T}\right)^{2}}{2\left(\frac{\sigma}{k_{\mathrm{B}} T}\right)^{2}-\varepsilon},
$$

where $\Delta E$ is a difference between delocalization energy $E_{\text {a }}$ and central energy $E_{0}$. Equation (4) can be solved numerically.

Effective recombination rate, $r_{\text {eff }}$, depends also on nonradiative recombination

$$
\begin{aligned}
& r_{\text {eff }}(E, T)=\frac{1}{\tau_{\mathrm{a}}+\alpha T \exp \left(-\varepsilon-\frac{\Delta E}{k_{\mathrm{B}} T}\right)} \\
& \quad+r_{\mathrm{nrad}} \exp \left(\frac{-E_{\mathrm{nrad}}}{k_{\mathrm{B}} T}\right),
\end{aligned}
$$

where $r_{\text {nrad }}$ is the nonradiative recombination rate and $E_{\text {nrad }}$ is thermal activation energy. Note that in formula (5), continuous delocalization of the carriers is assumed.

\section{Experiment}

Two samples, A and B, were used in our experiments.

Sample A was grown using standard metal organic chemical vapor deposition (MOCVD) technique. Its structure contained $1 \mu \mathrm{m}$ layer of $\mathrm{GaN}: \mathrm{Si}$, with silicon concentration equal to $3 \times 10^{18} / \mathrm{cm}^{3}$, then $40 \mathrm{~nm}$ thick $\mathrm{In}_{0.02} \mathrm{Ga}_{0.098} \mathrm{~N}$ buffer layer was grown, which was also $\mathrm{Si}$ doped with the same concentration. On top of this buffer layer, three $3.5 \mathrm{~nm} \mathrm{In}_{0.06} \mathrm{Ga}_{0.094} \mathrm{~N}$ quantum wells were grown, and all structure was covered by $20 \mathrm{~nm}$ GaN cap. This sample contained many regions in which photoluminescence energy changes in controlled way (see Ref. [12]).

Sample B was fabricated using molecular beam epitaxy (MBE) method and contained $110 \mathrm{~nm}$ thick layer of undoped GaN grown on gallium nitride template. On top of that layer, $\mathrm{Al}_{0.15} \mathrm{Ga}_{0.85} \mathrm{~N}$ alloy was grown of the thickness of $21 \mathrm{~nm}$, followed by $10 \mathrm{~nm}$ of GaN. Next layers were $\mathrm{Ga}_{0.89} \operatorname{In}_{0.11} \mathrm{~N}, \mathrm{Al}_{0.14} \mathrm{Ga}_{0.885} \operatorname{In}_{0.055} \mathrm{~N}$ and $\mathrm{Ga}_{0.93} \operatorname{In}_{0.07} \mathrm{~N}$ with thickness 40,18, and $18 \mathrm{~nm}$, respectively.

$\mathrm{PL}$ was measured in temperatures from $4 \mathrm{~K}$ to $300 \mathrm{~K}$ in continuous flow helium cryostat. The samples were excited by 0.1 ps pulses of the third harmonic of Ti:sapphire laser $(300 \mathrm{~nm})$. Temporal dependence of PL was measured with the use of a streak-camera.

The PL spectra (see Fig. 2) consisted of a few peaks. In both samples signal from exciton bound to donors could be seen at $3.47 \mathrm{eV}$, which is characteristic for GaN. Peaks from the main GaInN layers could be seen 

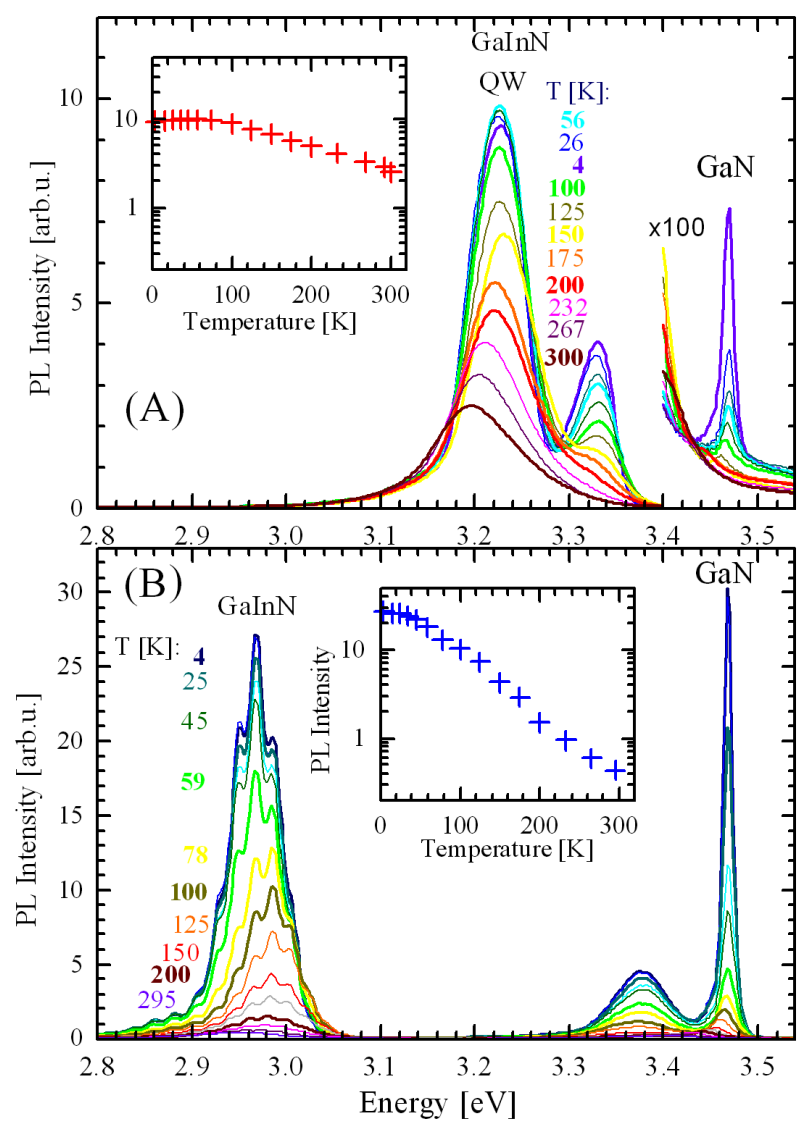

Fig. 2. GaInN/GaN spectra at different temperatures: (A) sample A and (B) sample B. Insets present intensities of GaInN peaks vs. temperature.

at energies $3.33 \mathrm{eV}$ and $2.96 \mathrm{eV}$ in samples $\mathrm{A}$ and $\mathrm{B}$, respectively. The full width at half-maximum for the QW peak of sample A was $70 \mathrm{meV}$ at helium temperature and it increased above $100 \mathrm{meV}$ at room temperature. Sample B showed strong interference fringes, so it was not possible to determine width of the peak.

It is visible that spectra change their intensity and energies. Intensity of GaN band to band emission decreases more than order of magnitude from 4 K to $300 \mathrm{~K}$. Similar changes of intensity are observed also for the sample $\mathrm{B}$, for all peaks. However, the intensity of GaInN QW emission in the sample A decreases only 4 times in room temperature. The energy of GaN peak decreases monotonically with temperature from $3.47 \mathrm{eV}$ to $3.41 \mathrm{eV}$ while peaks from GaInN layers reveal S-shape dependence.

For the sample A, in temperature range $4-150 \mathrm{~K}$ the energy decreases and increases back of about $5 \mathrm{meV}$. Sample B has better visible S-shape. Cooling from $120 \mathrm{~K}$ to $4 \mathrm{~K}$ causes energy decreases from $2.99 \mathrm{eV}$ to $2.97 \mathrm{eV}$. It is due to carriers diffusion to energy minima, enabled by long carrier lifetime in this temperature range. For the sample B lifetime becomes significantly longer during cooling below $100 \mathrm{~K}$ (see Fig. 3b).

The data present in Fig. 3 were analyzed on base of Eliseev's theory at $T>70 \mathrm{~K}$ and on the base of theory
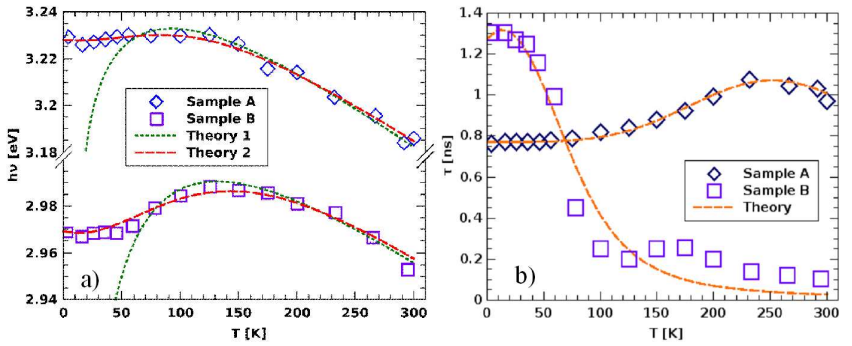

Fig. 3. (a) PL energy dependences on temperature for samples A and B. Experimental data are represented by squares, green dotted line represents theory of Eliseev and red dashed line comes from solving Eqs. (2) and (4).

(b) Luminescence decay time vs. temperature for sample A and for sample B. Orange dashed line represents fitting of Eq. (5).

proposed in this paper in full range. Equations (2), (4), and (5) were fitted at the same time with the common parameters. For energy and lifetime data sets, sum of least squares $\left(S_{E}^{2}\right.$ and $\left.S_{t}^{2}\right)$ were calculated and fitting procedure was realized by minimizing the length of vector of sums of least squares $\left(\sqrt{S_{E}^{2}+S_{t}^{2}}\right)$.

The values of parameters obtained during fitting procedures are shown in Table I.

TABLE I

Values of fitting parameters for samples A and B.

\begin{tabular}{c|c|c|c|c}
\hline \hline \multirow{2}{*}{} & \multicolumn{2}{|c|}{ Sample A } & \multicolumn{2}{c}{ Sample B } \\
\cline { 2 - 5 } & $\sigma[\mathrm{meV}]$ & $\Delta E[\mathrm{meV}]$ & $\sigma[\mathrm{meV}]$ & $\Delta E[\mathrm{meV}]$ \\
\hline Eliseev & 12 & - & 19 & - \\
\hline $\mathrm{Li}$ & 29 & 28 & 22 & 70 \\
\hline Our theory & 17 & 20 & 25.5 & 10
\end{tabular}

In the case of sample $\mathrm{A}$, there was significant radiative recombination, that dominated even in higher temperatures, which caused the growth of PL lifetime with temperature, as predicted by Eq. (3), in range up to $250 \mathrm{~K}$.

From analysis of curves plotted in Fig. 3 we can deduce that in sample A recombination is mainly radiative even at high temperature while sample B has strong nonradiative recombination starting already at low temperature $(50 \mathrm{~K})$. This result explains the observed behaviors of $\mathrm{PL}$ intensity in both samples. Sample A is bright up to high temperatures while sample B intensity decreases with temperature due to high nonradiative recombination rate.

\section{Conclusions}

We propose a model of recombination that involves effects of low-temperature excitons/carriers localization due to the potential fluctuations in quantum wells and layer of nitride semiconductors. This new model introduces the dependence of recombination time on energy and temperature, which are demonstrated by variety of experiments. The proposed model describes very well thermal dependences of both energy and recombination time observed in experiment performed on two samples. 
The two investigated samples have different proportions of radiative and non-radiative recombination. The deduced recombination properties described well temperature dependences of photoluminescence energy and exciton decay for the InGaN/GaN quantum wells and InAlGaN layer samples.

\section{Acknowledgments}

This work was partially supported by Polish National Science Centre within the Project DEC2013/11/B/ST3/04263.

\section{References}

[1] Y.P. Varshni, Physica 34, 149 (1967).

[2] L. Vińa, S. Logothetidis, M. Cardona, Phys. Rev. B 30, 1979 (1984).

[3] K.P. Korona, A. Wysmolek, K. Pakula, R. Stępniewski, J.M. Baranowski, I. Grzegory, B. Lucznik, M. Wróblewski, S. Porowski, Appl. Phys. Lett. 69 788 (1996).

[4] Y.-H. Cho, G.H. Gainer, A.J. Fischer, J.J. Song, S. Keller, U.K. Mishra, S.P. DenBaars, Appl. Phys. Lett. 73, 1370 (1998).
[5] M. Kunzer, C.-C. Leancu, M. Maier, K. Köhler, U. Kaufmann, J. Wagner, Phys. Status Solidi C 5, 2170 (2008)

[6] S.M. Olsthoorn, F.A.J.M. Driessen, A.P.A.M. Eijkelenboom, L.J. Giling, J. Appl. Phys. 73, 7798 (1993).

[7] A. Bell, S. Srinivasan, C. Plumlee, H. Omiya, F.A. Ponce, J. Christen, S. Tanaka, A. Fujioka, Y. Nakagawa, J. Appl. Phys. 95, 4670 (2004).

[8] P.G. Eliseev, J. Appl. Phys. 93, 5404 (2003).

[9] Q. Li, S.J. Xu, W.C. Cheng, M.H. Xie, S.Y. Tong, C.M. Che, H. Yang, Appl. Phys. Lett. 79, 1810 (2001).

[10] D. Rosales, T. Bretagnon, B. Gil, A. Kahouli, J. Brault, B. Damilano, J. Massies, M.V. Durnev, A.V. Kavokin, Phys. Rev. B 88, 125437 (2013).

[11] H. Gotoh, H. Ando, T. Takagahara, J. Appl. Phys. 81, 1785 (1997).

[12] P.A. Dróżdż, K.P. Korona, M. Sarzyński, T. Suski, R. Czernecki, D. Wasik, Phys. Status Solidi B 253, 284 (2016). 